\title{
Point-Set Embeddability of 2-Colored Trees
}

\author{
Fabrizio Frati ${ }^{1}$, Marc Glisse ${ }^{2}$, William J. Lenhart ${ }^{3}$, \\ Giuseppe Liotta ${ }^{4}$, Tamara Mchedlidze ${ }^{5}$, and Rahnuma Islam Nishat ${ }^{6}$ \\ 1 School of Information Technologies, The University of Sydney, Australia \\ ${ }^{2}$ INRIA Saclay - Ile-de-France, France \\ ${ }^{3}$ Computer Science Department, Williams College, U.S.A. \\ ${ }^{4}$ Dipartimento Ingegneria Elettronica e dell'Informazione, Universitá di Perugia, Italy \\ ${ }^{5}$ Institute of Theoretical Informatics, Karlsruhe Institute of Technology (KIT), Germany \\ ${ }^{6}$ Department of Computer Science, University of Victoria, Canada
}

\begin{abstract}
In this paper we study bichromatic point-set embeddings of 2-colored trees on 2-colored point sets, i.e., point-set embeddings of trees (whose vertices are colored red and blue) on point sets (whose points are colored red and blue) such that each red (blue) vertex is mapped to a red (resp. blue) point. We prove that deciding whether a given 2-colored tree admits a bichromatic point-set embedding on a given convex point set is an $\mathcal{N} \mathcal{P}$-complete problem; we also show that the same problem is linear-time solvable if the convex point set does not contain two consecutive points with the same color. Furthermore, we prove a $3 n / 2-O(1)$ lower bound and a $2 n$ upper bound $($ a $7 n / 6-O(\log n)$ lower bound and a $4 n / 3$ upper bound) on the minimum size of a universal point set for straight-line bichromatic embeddings of 2-colored trees (resp. 2-colored binary trees). Finally, we show that universal convex point sets with $n$ points exist for 1-bend bichromatic point-set embeddings of 2-colored trees.
\end{abstract}

\section{Introduction}

A point-set embedding of a planar graph $G$ on a point set $P$ is a mapping of each vertex of $G$ to a point in $P$ and of each edge of $G$ to a curve between its endpoints such that no two edges intersect. Point-set embeddability is a classical topic of research in Graph Drawing and a large number of results are known for several algorithmic and combinatorial problems related to point-set embeddings.

Gritzmann et al. [22] proved that a graph admits a straight-line point-set embedding on every point set in general position if and only if it is outerplanar. Straight-line embeddings of outerplanar graphs and trees on point sets in general position can be constructed efficiently [5|6]. On the other hand, deciding whether a planar graph admits a straight-line embedding on a given point set is $\mathcal{N} \mathcal{P}$-complete [8], even if the input planar graph is triconnected [3|17].

A particular attention has been devoted to determining the minimum size $f^{*}(n)$ of a universal point set for point-set embeddings of $n$-vertex planar graphs, i.e., a point set $P$ with $f^{*}(n)$ points such that every $n$-vertex planar graph $G$ admits a point-set embedding on an $n$-point subset of $P$. For straight-line point-set embeddings, the best known upper bound for $f^{*}(n)$ is $O\left(n^{2}\right)$ [10], while the best known lower bound is $\Omega(n)$ [928]. Concerning $k$-bend point-set embeddings, that are point-set embeddings

W. Didimo and M. Patrignani (Eds.): GD 2012, LNCS 7704, pp. 291-302, 2013.

(C) Springer-Verlag Berlin Heidelberg 2013 
in which each edge is allowed to bend $k$ times, Kaufmann and Wiese [27] proved that every point set with $n$ points is universal for 2-bend point-set embeddings of planar graphs, and that there exist a point set $P$ and a planar graph $G$ such that every point-set embedding of $G$ on $P$ has at least 2 bends on some edges (see also [13]). Moreover, Everett et al. proved in [19] that $f^{*}(n)=n$ for 1-bend point-set embeddings of planar graphs. Dujmović et al. [16] constructed small universal point sets $P$ that allow for point-set embeddings in which bends are also embedded at points of $P$.

Pach and Wenger [29] considered the point-set embeddability problem under the assumption that the mapping from the vertices of the graph to the points of the point set is given as part of the input. They showed that, for every point set in general position, a point set embedding with given mapping of any planar graph $G$ exists with $O(n)$ bends per edge and that $\Omega(n)$ bends per edge may be necessary even for paths.

Recently, the two variants of the point-set embedding problem (with or without mapping) have been unified by introducing the concept of $k$-colored point-set embedding, where the set of vertices and the set of points are colored with $k$ colors and each vertex is required to be drawn on a point with the same color [2|14|11|15]. Badent et al. [2] generalized the result by Pach and Wenger by proving that, for every $k \geq 2$, a $k$-colored planar graph admits a $k$-colored point-set embedding on every $k$-coloured set of points with $O(n)$ bends per edge and that $\Omega(n)$ bends may be necessary.

In this paper we study the 2 -colored point-set embeddability problem. That is, the vertices of the input graph $G$ and the points of the input point set $P$ are 2 -colored (i.e. each vertex of $G$ and each point of $P$ is either red or blue), and the point-set embedding of $G$ on $P$ requires red and blue vertices to be mapped to red and blue points, respectively. Such an embedding is called bichromatic point-set embedding. The coloring of the graph is called proper if no two adjacent vertices receive the same color.

In Section 3, we study the time complexity of deciding whether a 2-colored tree admits a bichromatic point-set embedding on a 2-colored point set. We show that the problem is $\mathcal{N} \mathcal{P}$-complete, even if the tree is properly 2 -colored and the point set is in convex position. Contrastingly with the $\mathcal{N} \mathcal{P}$-hardness result, we show that deciding whether a properly 2 -colored tree can be embedded on an alternating 2-colored convex point set is a linear-time solvable problem. An alternating point set is a convex point set such that no two consecutive points on the border of the convex hull have the same color.

In Section 4 we study the size $f(n)$ of the smallest universal 2-colored convex point set for bichromatic straight-line embeddings of 2 -colored $n$-vertex trees. Such a value is formally defined as follows: For any pair $(r, b)$ such that $r+b=n$, let $f(n, r, b)$ be the minimum number of points of a convex point set $P$ such that every $n$-vertex tree $T$ with $r$ red vertices and $b$ blue vertices admits a bichromatic point-set embedding on $P$. Then $f(n)=\max _{r, b: r+b=n} f(n, r, b)$. We show that $f(n) \leq 2 n$ and that $f(n) \geq 3 n / 2-O(1)$. Further, we show that, when restricting the attention to properly 2 -colored binary trees, we have $f(n) \leq 4 n / 3$ and $f(n) \geq 7 n / 6-O(\log n)$.

In Section 5, we show that allowing bends on the edges leads to have $f(n)=n$. Namely, we prove that every linearly-separable 2-colored point set (with $r$ red points and $b$ blue points) is universal for bichromatic 1-bend point-set embeddings of (possibly non-properly) 2-colored trees (with $r$ red vertices and $b$ blue vertices). 
Universal 2-colored point sets with $n$ points are known to exist for bichromatic straight-line point-set embeddings of several classes of 2-colored trees, namely properly 2-colored paths [1], (possibly non-properly) 2-colored paths [7], (possibly non-properly) 2-colored spider trees [18], forests with two connected components each containing exactly one red vertex [23|24], forests of star trees and forest of trees whose size differ by at most one [25]26], forests where each connected component contains exactly one red vertex [12], properly 2-colored trees whose leaves all have the same color [20], and (possibly non-properly) 2-colored rooted trees such that every vertex has at most one child having a color different from its own [20].

\section{Preliminaries}

In this paper we mostly adopt definitions of [14].

Let $T=(V, E)$ be a 2-colored tree. If $v$ is a vertex of $T$, we denote by $c(v)$ the color of $v$ and by $\bar{c}(v)$ the color different from $c(v)$. Let $T$ be a 2-colored tree $T$ which is rooted at one of its vertices. For a vertex $u$ of $T$, we denote by $T(u)$ the subtree of $T$ rooted at $u$. For a vertex $u$ of a rooted properly 2 -colored tree $T$, we define the surplus $s(T(u))$ of $T(u)$ inductively as follows: (1) if $u$ is a leaf of $T$, then $s(T(u))=1 ;(2)$ otherwise $s(T(u))=1-\sum_{w} s(T(w))$, where the sum is over all the children $w$ of $u$. Hence, $s(T(u))$ is equal to the number of vertices of color $c(u)$ minus the number of vertices of color $\bar{c}(u)$ in $T(u)$. A rooted properly 2-colored tree $T$ is balanced if $|s(T(u))| \leq 1$, for every vertex $u$ of $T$. A (non-rooted) properly 2 -colored tree is balanced if it can be rooted so that the resulting rooted tree is balanced. A rooted properly 2-colored tree $T$ is strongly balanced if $0 \leq s(T(u)) \leq 1$, for every vertex $u$ of $T$.

A planar point set is a convex point set if none of its points lies in the convex hull of the others. Given a point $p$ in a 2 -colored point set, we denote by $c(p)$ the color of $p$. The next lemma explains the structure of an embedding of a tree on a convex point set.

Lemma 1 ([4]). Let $T$ be any $n$-vertex tree and let $P$ be any convex point set of size $n$. Let $u$ be any vertex of $T$ and let $T_{1}, T_{2}, \ldots, T_{k}$ be the subtrees of $T$ obtained by removing $u$ and its incident edges from $T$. In any straight-line embedding of $T$ on $P$, the vertices of $T_{i}$ are mapped on a set of consecutive points of $P$, for each $i=1,2, \ldots, k$.

Given a 2-colored point set $P$ and a line $l$, we denote by $\sigma_{P}^{l}$ the sequence of blue and red points obtained by the orthogonal projection of $P$ on $l$. An $h$-page book embedding of a graph $G$ is a drawing of $G$ such that all the vertices of $G$ are drawn as points on a straight line $\Lambda$ called spine, each edge of $G$ is drawn on one of $h$ half-planes, called pages, having $\Lambda$ as a common boundary, and no two edges in the same page cross. Let $\sigma$ be a sequence of red and blue points on a line $\Lambda$. An $h$-page book embedding of a graph $G$ is consistent with $\sigma$ if each vertex $v$ of $G$ is represented by a point $p$ of $\sigma$ and $c(v)=c(p)$. Notice that the exact position of the points of $\sigma$ on $\Lambda$ is not relevant for the existence of the book embedding, and only their relative order is important. Let $\Gamma$ be an $h$-page book embedding of a graph $G$. A vertex $v$ of $G$ is called accessible from a page $\pi$ if there is no edge $(u, w)$ in $\pi$ such that $u<v<w$ in the linear ordering of $\Gamma$. Two vertices accessible from a common page can be connected by an edge drawn in that page without creating any crossings. A book embedding $\Gamma$ of a 2 -colored tree is 
called alternating if the colors of the vertices along the spine of $\Gamma$ alternate. In such an embedding, $l(\Gamma)$ and $r(\Gamma)$ refer to the two end-vertices of the embedding.

Generalizing a previous result presented in [27] for non-colored graphs, the following statement was proved by Di Giacomo et al. in [14].

Theorem 1 ([14]). Let $G=(V, E)$ be a 2-colored graph and $P$ be a 2-colored point set, with $|P|=|V|$. G admits 1-bend bichromatic point-set embedding on $P$ if and only there exists a line $l$ such that $G$ admits a 2-page book embedding consistent with $\sigma_{P}^{l}$.

The following result will be used in the following sections.

Theorem 2 (Folklore). Let $G$ be an n-vertex graph. $G$ has a 1-page book embedding if and only if $G$ has a straight-line point-set embedding on an n-point convex point set.

\section{Deciding Bichromatic Straight-Line Embeddability}

In this section we consider the computational complexity of the problem of deciding whether a properly 2 -colored tree can be embedded on a 2 -colored convex point set. We start by showing that, in general, such a problem is $\mathcal{N} \mathcal{P}$-complete.

Theorem 3. Given a n-vertex properly 2-colored tree $T$ and a 2-colored convex point set $P$ with $n$ points, it is $\mathcal{N} \mathcal{P}$-complete to decide whether $T$ admits a bichromatic straight-line point-set embedding on $P$.

In the following we prove Theorem 3 . First, the problem is trivially in $\mathcal{N} \mathcal{P}$. In order to prove $\mathcal{N} \mathcal{P}$-hardness, we construct a reduction from the 3 -Partition problem, which is defined as follows. Given a bound $B \in \mathbb{Z}^{+}$and given a set $A=\left\{a_{1}, \ldots, a_{3 m}\right\}$ with $a_{i} \in \mathbb{Z}^{+}$such that $\frac{B}{4}<a_{i}<\frac{B}{2}$, is it possible to construct $m$ disjoint sets $A_{1}, \ldots, A_{m} \subset A$, with $\left|A_{i}\right|=3$ and with $\sum_{a \in A_{i}} a=B$, for each $1 \leq i \leq m$ ?

We use the fact that 3 -Partition is $\mathcal{N} \mathcal{P}$-hard even if $B$ is bounded by a polynomial in $m[21]$ and if the numbers $A=\left\{a_{1}, \ldots, a_{3 m}\right\}$ are all even. Let $(A, B)$ be an instance of 3-Partition. We show how to construct an equivalent instance $(T, P)$ of bichromatic straight-line point-set embeddability.

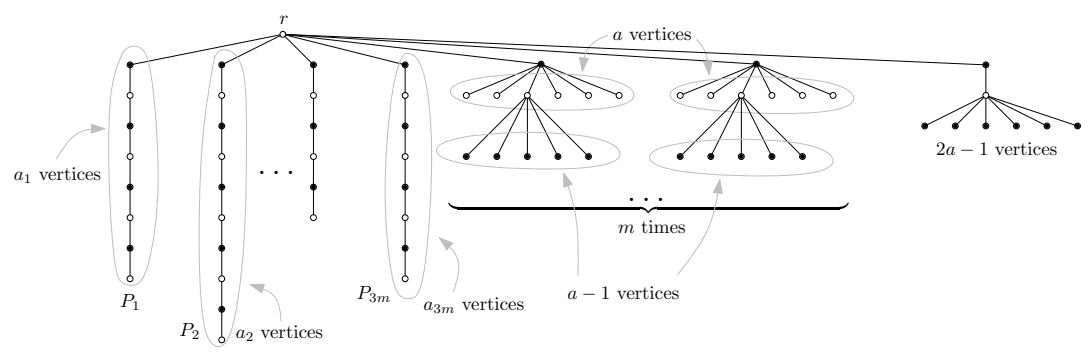

Fig. 1. 2-colored tree $T$ for the proof of $\mathcal{N} \mathcal{P}$-hardness 


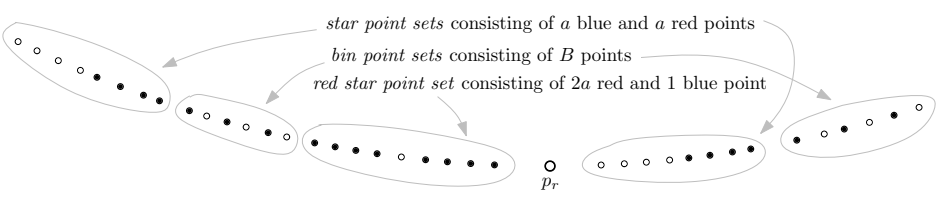

Fig. 2. 2-colored point set $P$ for the proof of $\mathcal{N} \mathcal{P}$-hardness

Tree $T$ is constructed as follows (see Fig. 1). Let $r$ be a blue vertex of $T$, which we set to be the root of $T$. For each $a_{i}, 1 \leq i \leq 3 \mathrm{~m}$, we add to $T$ an alternating red-blue path $P_{i}$ with $a_{i}$ vertices and connect its red end-vertex with $r$. Let $a=\max _{1 \leq i \leq 3 m} a_{i}$. We further add $m$ subtrees to $T$. Each of such subtrees is rooted at a red vertex, has $a$ blue children, and one of these blue children has $a-1$ red children. We call such subtrees star subtrees. Finally, we add to $T$ a subtree rooted at a red vertex having one blue child, which in turn has $2 a-1$ red children. We call this subtree red subtree.

Point set $P$ is constructed as follows (see Fig. 2). Let a star point set be a convex point set consisting of $a$ blue points followed by $a$ red points. Let a red star point set be a convex point set that contains $a$ red points, then one blue point, and then $a$ red points. Let a bin point set be a convex point set consisting of $B$ alternating points starting with a red point. Let $P$ be a convex point set that contains one red star point set, then a blue point $p_{r}$, and then $m$ star point sets and $m$ bin point sets, appearing in an alternate manner, and starting with a star point set. Note that $p_{r}$ is adjacent to a red star point set from one side and to a star point set from the other side. We have the following.

Lemma 2. $(A, B)$ is a positive instance of 3-Partition if and only if T admits a bichromatic straight-line point-set embedding on $P$.

Proof sketch: By Lemma1 in any bichromatic straight-line point-set embedding of $T$ on $P$, the red subtree is mapped to the red star point set, and each star subtree is mapped to a distinct star point set. The remaining bin point sets are alternating point sets each with $B$ points. Again by Lemma 1 each bichromatic straight-line point-set embedding of $T$ on $P$ maps three paths $P_{i}, P_{j}$, and $P_{k}$ on a single bin point set. This is possible if and only if $a_{i}+a_{j}+a_{k}=B$.

The described reduction from $(A, B)$ to $(T, P)$ is polynomial, given that $B$ is bounded by a polynomial in $m$. Hence, Lemma 2 completes the proof of Theorem 3 .

Contrastingly with Theorem 3 , we show that deciding whether a properly 2-colored tree can be embedded on an alternating 2-colored convex point set is a linear-time solvable problem. Namely, in Lemmata 3 and 4 we show that a properly 2 -colored tree admits a bichromatic straight-line embedding on an alternating point set if and only if it is balanced. Moreover, in Theorem 4 we show how to test in linear time whether a tree is balanced.

Lemma 3. Every properly 2-colored rooted balanced tree $T$ with $n$ vertices admits a bichromatic straight-line embedding on any alternating point set $P$ with $n$ points.

Proof sketch: The following claim is proved. For any vertex $v$ of $T$, tree $T(v)$ admits an alternating 1-page book embedding $\Gamma$ such that $v$ is accessible and such that: $(\mathrm{C} 1)$ 
If $s(T(v))=1$, then $c(v)=c(l(\Gamma))=c(r(\Gamma))$; (C2) if $s(T(v))=0$, then $c(l(\Gamma)) \neq$ $c(r(\Gamma))$; and (C3) if $s(T(v))=-1$, then $c(v) \neq c(l(\Gamma))=c(r(\Gamma))$. The claim, together with Theorem 2 , implies the lemma.

The proof of the claim is by induction on $|T(v)|$. The claim is trivially proved if $|T(v)|=1$. If $|T(v)|>1$, inductively constructed 1-page book embeddings of the subtrees of $T$ rooted at the children $v_{1}, v_{2}, \ldots, v_{k}$ of $v$ are combined with $v$ to construct a 1-page book embedding $\Gamma$ of $T(v)$ in such a way that $v$ is accessible and that (C1)(C3) are satisfied. Such a construction distinguishes the cases in which $s(T(v))=1$, $s(T(v))=0$, or $s(T(v))=-1$. In each case, subtrees with surplus $-1,0$, and 1 are suitably alternated in order to guarantee that $\Gamma$ is alternating and that $(\mathrm{C} 1)-(\mathrm{C} 3)$ are satisfied; moreover, the accessibility of $v_{1}, v_{2}, \ldots, v_{k}$ ensures the planarity of $\Gamma$.

Lemma 4. Suppose that an $n$-vertex properly 2 -colored tree $T$ admits a bichromatic straight-line embedding on an n-point alternating point set. Then $T$ is balanced.

Proof: If $T$ is not balanced then, for every choice of the root of $T$, there exists a vertex $u$ such that $s(T(u))>1$ or $s(T(u))<-1$. By Lemma11 in any point-set embedding of $T$ on $P, T(u)$ is embedded on a set of consecutive points of $P$. However, in any set of $|T(u)|$ consecutive points of $P$, the number of red points and the number of blue points differ at most by one. Hence, in any point-set embedding of $T$ on $P$, a red vertex is mapped to a blue point or a blue vertex is mapped to a red point.

Theorem 4. Let $T$ be an $n$-vertex properly 2-colored tree and let $P$ be an $n$-point alternating 2-colored convex point set. It can be decided in $O(n)$ time whether $T$ admits a bichromatic straight-line point-set embedding on $P$ or not.

Proof sketch: By Lemmata 3 and 4, $T$ admits a bichromatic straight-line point-set embedding on $P$ if and only if it is balanced. Moreover, it can be tested in $O(n)$ time whether $T$ is balanced or not as follows. First, $T$ is rooted at an arbitrary vertex $v$ and a test is performed in $O(n)$ time determining whether $T$ is balanced with $v$ as the root. Such a test consists of a simple bottom-up traversal of $T$. If the test succeeds, then $T$ is balanced. Otherwise, if there exist two vertices $u_{1}$ and $u_{2}$ such that (1) $\left|s\left(T\left(u_{1}\right)\right)\right|>1$, (2) $\left|s\left(T\left(u_{2}\right)\right)\right|>1$, (3) $u_{1}$ is neither an ancestor nor a descendant of $u_{2}$, then $T$ is not balanced. Otherwise, all the vertices $u$ such that $|s(T(u))|>1$ lie in the same path from $v$ to a leaf. Let $u^{\prime}$ be the one of such vertices that is farthest from $v$. If any vertex $u^{*}$ exists such that $T$ is balanced if rooted at $u^{*}$, then $u^{*}$ is in $T\left(u^{\prime}\right)$. Let $T_{p}$ be the subtree of $T$ obtained by deleting the vertices in $T\left(u^{\prime}\right)$ and their incident edges from $T$. Root $T_{p}$ at the only vertex $p$ that is neighbor of $u^{\prime}$ in $T$. Test in $O(n)$ time if $T_{p}$ is balanced with $p$ as a root. If not, we can conclude that $T$ is not balanced, otherwise we have that $T$ is balanced with $u^{\prime}$ as a root. This concludes the test and the proof of the theorem.

\section{Universal Point Sets for Bichromatic Straight-Line Embeddings}

In this section we consider the problem of determining the smallest value $f(n)$ such that there exists a universal 2-colored convex point set with $f(n)$ points for bichromatic straight-line embeddings of $n$-vertex 2 -colored trees. In Theorem 5 we show that $f(n) \leq 2 n$; moreover, in Theorem 6 we show that $f(n) \geq 3 n / 2-O(1)$. 


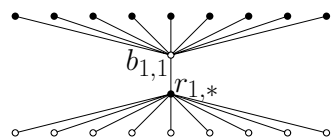

(a)

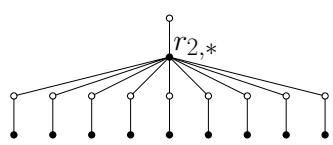

(b)

Fig. 3. (a) Tree $T_{1}$. (b) Tree $T_{2}$.

Theorem 5. Any $2 n$-point alternating convex point set is universal for bichromatic straight-line embeddings of 2-colored $n$-vertex trees.

Proof sketch: Let $P$ be any $2 n$-point alternating convex point set and let $T$ be any arbitrarily-rooted $n$-vertex 2 -colored tree. The following claim is proved. For any subtree $S$ of $T$ and any subset $P^{\prime}$ of $2|S|$ consecutive points of $P$, there exists a bichromatic straight-line embedding of $S$ on $P^{\prime}$ satisfying the following property: (S1) The root $s$ of $S$ is mapped on one of the "first two points" of $P^{\prime}$, and no vertex of $S$ different from $s$ is mapped on any of such two points. The claim implies the theorem with $S=T$. The proof is by induction on $|S|$. If $|S|=1$, then the claim is trivially true. If $|S|>1$, then a bichromatic straight-line embedding of $S$ on $P^{\prime}$ can be constructed by placing $s$ on one of the first two points of $P^{\prime}$, depending on the color of $s$, and by inductively constructing bichromatic straight-line embeddings of the subtrees $S_{1}, S_{2}, \ldots, S_{l}$ of $s$ on subsets of $P$ consisting of $2\left|S_{1}\right|, 2\left|S_{2}\right|, \ldots, 2\left|S_{l}\right|$ consecutive points, respectively. Property (S1) for the embeddings of $S_{1}, S_{2}, \ldots, S_{l}$ ensures the planarity of the embedding of $S$.

Next, we present two properly 2 -colored trees $T_{1}$ and $T_{2}$, each with $n / 2$ red vertices and $n / 2$ blue vertices, such that any 2 -colored convex point set $P$ such that both $T_{1}$ and $T_{2}$ admit bichromatic straight-line embeddings on $P$ has at least $3 n / 2-O(1)$ points. Tree $T_{1}$ has one red vertex $r_{1, *}$ that is connected to $n / 2$ blue vertices $b_{1,1}, \ldots, b_{1, n / 2}$. Vertices $b_{1,2}, \ldots, b_{1, n / 2}$ are leaves, while $b_{1,1}$ is also connected to $n / 2-1$ red vertices $r_{1,1}, \ldots, r_{1, n / 2-1}$ that are leaves. See Fig. 3.a). Tree $T_{2}$ has one red vertex $r_{2, *}$ that is connected to $n / 2$ blue vertices $b_{2,1}, \ldots, b_{2, n / 2}$. Each blue vertex $b_{2, i}$, for $2 \leq i \leq n / 2$, is connected to a red vertex $r_{2, i}$ which is a leaf. See Fig. 3. (b).

Lemma 5. Any convex point set $P$ such that both $T_{1}$ and $T_{2}$ admit bichromatic straightline embeddings on $P$ has at least $\frac{3 n}{2}-O(1)$ points.

Proof sketch: The main idea that allows us to prove the lemma is the one of partial discrepancy of a 2-colored convex point set. Let $P=R \cup B$ be a 2-colored convex point set, where $R$ and $B$ denote the sets of red and blue points in $P$, respectively. We define the discrepancy of $P$ as $|R-B|$. The partial discrepancy of $P$ is the maximum discrepancy of any point set which is composed of consecutive points of $P$. By Lemma 1 we have that any 2-colored convex point set $P_{1}$ with $\left|P_{1}\right|=n$ such that $T_{1}$ admits a bichromatic straight-line embedding on $P_{1}$ is composed of two sequences of consecutive points, each one with discrepancy $n / 2-1$. Again by Lemma 1 , we have that any 2-colored convex point set $P_{2}$ with $\left|P_{2}\right|=n$ such that $T_{2}$ admits a bichromatic straight-line embedding on $P_{2}$ has partial discrepancy at most 3 . Because of such two properties, if any straight-line embedding of $T_{2}$ maps $k_{A}$ red vertices of $T_{2}$ on red points in $P_{1}$, the same embedding also maps $k_{A}-O(1)$ blue vertices of $T_{2}$ on points 
not in $P_{1}$. Analogously, if any straight-line embedding of $T_{2}$ maps $k_{B}$ blue vertices of $T_{2}$ on blue points in $P_{1}$, the same embedding also maps $k_{B}-O(1)$ red vertices of $T_{2}$ on points not in $P_{1}$. Such considerations lead to prove the bound in the lemma.

We get the following theorem.

Theorem 6. Any 2-colored convex point set which is universal for bichromatic straightline point-set embeddings of 2-colored n-vertex trees has at least $3 n / 2-O(1)$ points.

We now restrict our attention to properly 2-colored binary trees. We denote by $g(n)$ the smallest value such that there exists a universal 2-colored convex point set with $g(n)$ points for bichromatic straight-line embeddings of properly 2 -colored $n$-vertex binary trees. We provide an upper and a lower bound for $g(n)$. In Theorem 7 we show that $g(n) \leq 4 n / 3$; moreover, in Theorem 8 we show that $g(n) \geq 7 n / 6-O(\log n)$.

Theorem 7. Any 4n/3-point alternating convex point set is universal for bichromatic straight-line embeddings of n-vertex properly 2-colored binary trees.

The proof of Theorem 7 uses two ingredients. The first one is Lemma 3, stating that every $n$-vertex properly 2 -colored balanced tree admits a bichromatic straight-line embedding on any $n$-point alternating point set; the second one is Lemma 6 , stating that every $n$-vertex properly 2 -colored binary tree is a subtree of a properly 2 -colored strongly balanced tree with $4 n / 3$ vertices. The two results directly imply Theorem 7 .

Lemma 6. Let $T$ be an $n$-vertex properly 2-colored binary tree. There exists a properly 2 -colored strongly balanced tree $T^{\prime}$ with $4 n / 3$ vertices such that $T$ is a subtree of $T^{\prime}$.

Proof sketch: We make $T$ strongly balanced in a bottom-up way. The subtrees rooted at the leaves of $T$ are strongly balanced. Consider any internal vertex $v$ of $T$ such that all the subtrees rooted at the descendants of $v$ are strongly balanced. W.l.o.g. assume that $v$ is red. We have that $T(v)$ is not strongly balanced if and only if the subtrees $T\left(u_{1}\right)$ and $T\left(u_{2}\right)$ rooted at the children $u_{1}$ and $u_{2}$ of $v$ have surplus 1 . In this case, we insert a red vertex in $T\left(u_{1}\right)$ as follows. If $u_{1}$ has no child, then it suffices to insert a red vertex as a child of $u_{1}$. If $u_{1}$ has at least one child $w$ (which has color red), we have that $T(w)$ has surplus 0 , hence $w$ has at least one child $z$ which has color blue and surplus 1 . We insert the new red vertex in $T(z)$. Eventually this procedure inserts a red vertex in a tree with exactly one blue vertex and no red vertices. After such an insertion $T(v)$ is strongly balanced. Whenever the above described procedure inserts a vertex to make a subtree $T(v)$ strongly balanced we have that $T(v)$ has surplus 0 . Moreover, $T(v)$ is not strongly balanced if and only if both $T\left(u_{1}\right)$ and $T\left(u_{2}\right)$ have surplus 1 . Hence, if a vertex has to be inserted to make $T(v)$ strongly balanced, then $v$ has exactly two children $u_{1}$ and $u_{2}$ and no vertex was inserted in order to make $T\left(u_{1}\right)$ and $T\left(u_{2}\right)$ strongly balanced. The number of inserted vertices is thus at most $n / 3$.

Next, we show a lower bound of $7 n / 6-O(\log n)$ for $g(n)$. Namely, we present two properly 2-colored binary trees $T_{1}$ and $T_{2}$, each with $n / 2$ red vertices and $n / 2$ blue vertices, such that any convex point set $P$ such that both $T_{1}$ and $T_{2}$ admit bichromatic straight-line embeddings on $P$ has at least $7 n / 6-O(\log n)$ points.

Tree $T_{1}$ is a balanced binary tree. In order to formally define $T_{1}$, we define a more general class $T(c, m)$ of trees as follows. The root of $T(c, m)$ has color $c \in\{$ red, blue $\}$ 


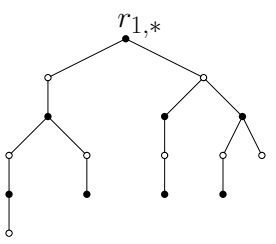

(a)

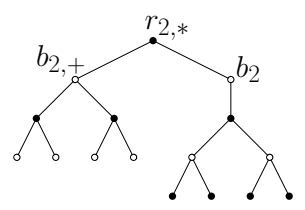

(b)

Fig. 4. (a) Binary tree $T_{1}$. (b) Binary tree $T_{2}$.

and $T(c, m)$ has $m$ vertices, for some $m$ even; if $m=2$, then the root $r(T(c, m))$ of $T(c, m)$ has one child with color $c^{\prime} \neq c$. If $m>2$, then $r(T(c, m))$ has two children $t_{x}$ and $t_{y}$, both with color $c^{\prime} \neq c$. Vertex $t_{x}$ has exactly one child $t_{z}$ with color $c$. If $m / 2$ is odd, then $t_{y}$ is the root of a tree $T\left(c^{\prime}, \frac{m-2}{2}\right)$ and $t_{z}$ is the root of a tree $T\left(c, \frac{m-2}{2}\right)$, while if $m / 2$ is even, then $t_{y}$ is the root of a tree $T\left(c^{\prime}, \frac{m}{2}\right)$ and $t_{z}$ is the root of a tree $T\left(c, \frac{m-4}{2}\right)$. Finally, we define $T_{1}=T($ red, $n)$, with $r(T($ red, $n))=r_{1, *}$. See Fig. 4(a).

Tree $T_{2}$ is rooted at a red vertex $r_{2, *}$ that has two blue vertices $b_{2,+}$ and $b_{2}$ as children; vertex $b_{2,+}$ is the root of a complete binary tree $T_{b}$ whose height (i.e. the number of edges in the longest path from the root to a leaf) is $h=\log _{2}(n)-2$; vertex $b_{2}$ is connected to a red vertex $r_{2,+}$ which is the root of a complete binary tree $T_{r}$ whose height is also $h$. We only consider the cases in which $h$ is even. See Fig. 4(b).

Lemma 7. Any 2-colored convex point set $P$ such that both $T_{1}$ and $T_{2}$ admit bichromatic straight-line embeddings on $P$ has at least $\frac{7 n}{6}-O(\log n)$ points.

Proof sketch: As in the proof of Lemma 5, we exploit the concept of discrepancy of a point set. By Lemma1, any 2-colored convex point set $P_{2}$ with $\left|P_{2}\right|=n$ such that $T_{2}$ admits a bichromatic straight-line embedding on $P_{2}$ is composed of two sequences of consecutive points, each one with discrepancy larger than $n / 6$. Moreover, any 2-colored convex point set $P_{1}$ with $\left|P_{1}\right|=n$ such that $T_{1}$ admits a bichromatic straight-line embedding on $P_{1}$ has $O(\log n)$ partial discrepancy. In order to prove the last statement, we introduce the concept of right discrepancy of a point set $P$ as the maximum discrepancy among all subsets of $P$ that can be obtained by walking clockwise starting from a designated point (representing the position of the root of $T_{1}$ ). The left discrepancy is defined analogously (with clockwise replaced by counterclockwise). Then, we prove that: (1) The left and right discrepancy of any 2 -colored convex point set $P(c, m)$ with $m$ points such that $T(c, m)$ admits a bichromatic straight-line embedding on $P(c, m)$ are at most $2 h(T(c, m))+1$, where $h(T(c, m))$ is the height of $T(c, m)$ (the proof is by induction on $h(T(c, m))$; (2) the discrepancy of any 2-colored convex point set $P(c, m)$ with $m$ points such that $T(c, m)$ admits a bichromatic straight-line embedding on $P(c, m)$ is at most $4 h(T(c, m))+1$ (the proof exploits the fact that the discrepancy of $P(c, m)$ is at most the sum of the left discrepancy and of the right discrepancy of two subtrees of $T(c, m))$; and (3) $h(T(c, m)) \leq 1+2 \log _{2} m$. The last two statements imply that $P_{1}$ has partial discrepancy at most $5+8 \log _{2} n$. The reminder of the proof is similar to the proof of Lemma 5 that is, given that $P_{2}$ is composed of two sequences of consecutive points, each one with discrepancy larger than $n / 6$, and given that $P_{1}$ has 
$O(\log n)$ partial discrepancy, we have that $n / 6-O(\log n)$ points of $P_{1}$ do not belong to $P_{2}$, thus leading to the $7 n / 6-O(\log n)$ lower bound for the size of any point set on which $P_{1}$ and $P_{2}$ can be both embedded.

We thus obtain the following.

Theorem 8. Any 2-colored convex point set which is universal for bichromatic pointset embeddings of properly 2 -colored $n$-vertex binary trees has at least $7 n / 6-O(\log n)$ points.

\section{Universal Point Sets for Bichromatic 1-Bend Embeddings}

In this section we study how allowing bends on the edges affects the size of universal point sets for bichromatic point-set embeddings of 2-colored trees. We prove that there exist (even convex) $n$-point universal point sets for 1-bend point-set embeddings of (even non-properly) 2-colored trees. More in general, we show that every 2-colored tree admits a 1-bend point-set embedding on every linearly separable point set (which is a point set such that there exists a line separating the red points from the blue points).

In order to do that we first prove that every 2 -colored tree admits an $(r, b)$-separable book embedding, i.e., a 2-page book embedding where all the blue vertices appear on the spine before all the red vertices.

Lemma 8. Every 2-colored tree admits an $(r, b)$-separable 2-page book embedding.

Proof sketch: The proof considers the maximal monochromatic connected subgraphs of $T$, in the following called monochromatic subtrees of $T$, and embeds such monochromatic subtrees in a breadth-first search ordering. That is, at the first step any monochromatic subtree is embedded. At step $i$, with $i \geq 2$, all the monochromatic subtrees having vertices adjacent to monochromatic subtrees embedded at step $i-1$ are embedded. Observe that all the vertices of the monochromatic subtrees embedded at step $i$ have the same color, for each $i \geq 1$. The book embedding is constructed in such a way that all the edges that are embedded at step $i$ lie on the same page; moreover, no two edges embedded at consecutive steps lie on the same page. Furthermore, vertices embedded at step $i$ are embedded either to the right or to the left of all the vertices embedded before step $i$.

We get the following.

Theorem 9. Let $T$ be a 2-colored tree with $r$ red vertices and b blue vertices, and let $P$ be a linearly separable point set with $r$ red and $b$ blue points. Then $T$ admits a 1-bend bichromatic embedding on $P$.

Proof: Consider the point set $P$. Let $l$ be a line in the plane that separates the blue points from the red points and that is not parallel to any line defined by two points of $P$. Let $f$ be a line perpendicular to $l$. Consider the sequence $\sigma_{P}^{f}$. By Theorem $9, T$ admits a 2-page book embedding consistent with $\sigma_{P}^{f}$. Therefore, by Theorem $1, T$ admits a bichromatic 1-bend point-set embedding on $P$. 


\section{Conclusions and Open Problems}

In this paper we have shown several algorithmic and combinatorial results related to the point-set embeddability of 2-colored trees on 2 -colored point sets.

We have shown that testing the bichromatic point-set embeddability of a 2-colored tree on a convex point set is an $\mathcal{N} \mathcal{P}$-complete problem. It would be interesting to study whether this problem becomes polynomial-time solvable when the input is a 2-colored binary tree. We suspect that an $\mathcal{N} \mathcal{P}$-hardness proof similar to the one we presented could be designed even in this restricted case; however, we defer such an intuition to further research. Further, we have proved upper and lower bounds for the minimum size of a universal convex point set for bichromatic point-set embeddings of 2 -colored trees and 2-colored binary trees. Tightening these bounds is a natural research direction.

The most interesting open problems are, in our opinion, related to the bichromatic point-set embeddability on point sets in general position. E.g., do point sets in general position with less than $2 n$ points exist which are universal for bichromatic point-set embeddings of $n$-vertex 2 -colored trees? Do universal point sets for bichromatic pointset embeddings of $n$-vertex 2-colored planar graphs require $\omega(n)$ points? An $O\left(n^{2}\right)$ upper bound for the last question can be proved by considering an $O(n) \times O(n)$ grid and by replacing each grid point $p$ with one red and one blue point close to $p$. Wellknown straight-line grid drawing algorithms (e.g. [10]) provide the claimed bound.

Acknowledgments. The authors thank Henk Meijer for fruitful discussions on the problems considered in this paper. This research was initiated at the International INRIAMcGill-Victoria Workshop on Problems in Computational Geometry, which was held at the Bellairs Research Institute of McGill University in Barbados. The research in this paper was partially supported by MIUR of Italy under project AlgoDEEP prot. 2008TFBWL4.

\section{References}

1. Abellanas, M., Garcia-Lopez, J., Hernández-Peñalver, G., Noy, M., Ramos, P.A.: Bipartite embeddings of trees in the plane. Discr. Appl. Math. 93(2-3), 141-148 (1999)

2. Badent, M., Di Giacomo, E., Liotta, G.: Drawing colored graphs on colored points. Theor. Comp. Sci. 408(2-3), 129-142 (2008)

3. Biedl, T., Vatshelle, M.: The point-set embeddability problem for plane graphs. In: SoCG 2012 (2012) (to appear)

4. Binucci, C., Di Giacomo, E., Didimo, W., Estrella-Balderrama, A., Frati, F., Kobourov, S.G., Liotta, G.: Upward straight-line embeddings of directed graphs into point sets. Computat. Geom. Th. Appl. 43, 219-232 (2010)

5. Bose, P.: On embedding an outer-planar graph in a point set. Comput. Geom. Th. Appl. 23(3), 303-312 (2002)

6. Bose, P., McAllister, M., Snoeyink, J.: Optimal algorithms to embed trees in a point set. J. Graph Alg. Appl. 1(2), 1-15 (1997)

7. Brandes, U., Erten, C., Estrella-Balderrama, A., Fowler, J.J., Frati, F., Geyer, M., Gutwenger, C., Hong, S., Kaufmann, M., Kobourov, S.G., Liotta, G., Mutzel, P., Symvonis, A.: Colored simultaneous geometric embeddings and universal pointsets. Algorithmica 60(3), 569-592 (2011) 
8. Cabello, S.: Planar embeddability of the vertices of a graph using a fixed point set is NP-hard. J. Graph Algorithms Appl. 10(2), 353-366 (2006)

9. Chrobak, M., Karloff, H.: A lower bound on the size of universal sets for planar graphs. SIGACT News 20, 83-86 (1989)

10. de Fraysseix, H., Pach, J., Pollack, R.: How to draw a planar graph on a grid. Combinatorica 10, 41-51 (1990)

11. Di Giacomo, E., Didimo, W., Liotta, G., Meijer, H., Trotta, F., Wismath, S.K.: k-colored point-set embeddability of outerplanar graphs. J. Graph Alg. Appl. 12(1), 29-49 (2008)

12. Di Giacomo, E., Didimo, W., Liotta, G., Meijer, H., Wismath, S.K.: Point-set embeddings of trees with given partial drawings. Comput. Geom. 42(6-7), 664-676 (2009)

13. Di Giacomo, E., Didimo, W., Liotta, G., Wismath, S.: Curve-constrained drawings of planar graphs. Comput. Geom. Theory Appl. 30, 1-23 (2005)

14. Di Giacomo, E., Liotta, G., Trotta, F.: On embedding a graph on two sets of points. Int. J. of Found. Comp. Sci. 17(5), 1071-1094 (2006)

15. Di Giacomo, E., Liotta, G., Trotta, F.: Drawing colored graphs with constrained vertex positions and few bends per edge. Algorithmica 57, 796-818 (2010)

16. Dujmovic, V., Evans, W., Lazard, S., Lenhart, W., Liotta, G., Rappaport, D., Wismath, S.: On Point-Sets That Support Planar Graphs. In: van Kreveld, M., Speckmann, B. (eds.) GD 2011. LNCS, vol. 7034, pp. 64-74. Springer, Heidelberg (2012)

17. Durocher, S., Mondal, D.: On the Hardness of Point-Set Embeddability (Extended Abstract). In: Rahman, M. S., Nakano, S.-I. (eds.) WALCOM 2012. LNCS, vol. 7157, pp. 148-159. Springer, Heidelberg (2012)

18. Estrella-Balderrama, A., Fowler, J.J., Kobourov, S.G.: Colored simultaneous geometric embeddings and universal pointsets. In: CCCG 2009, pp. 17-20 (2009)

19. Everett, H., Lazard, S., Liotta, G., Wismath, S.K.: Universal sets of $n$ points for one-bend drawings of planar graphs with $n$ vertices. Discr. Comp. Geom. 43(2), 272-288 (2010)

20. van Garderen, M., Liotta, G., Meijer, H.: Universal point sets for 2-coloured trees. Inf. Process. Lett. 112(8-9), 346-350 (2012)

21. Garey, M., Johnson, D.: Computers and Intractability: A Guide to the Theory of NPCompleteness. W. H. Freeman \& Co., New York (1979)

22. Gritzmann, P., Mohar, B., Pach, J., Pollack, R.: Embedding a planar triangulation with vertices at specified positions. The American Mathematical Monthly 98, 165-166 (1991)

23. Ikebe, Y., Perles, M.A., Tamura, A., Tokunaga, S.: The rooted tree embedding problem into points in the plane. Discr. Comp. Geom. 11, 51-63 (1994)

24. Kaneko, A., Kano, M.: Straight-line embeddings of two rooted trees in the plane. Discr. Comp. Geom. 21(4), 603-613 (1999)

25. Kaneko, A., Kano, M.: Straight line embeddings of rooted star forests in the plane. Discr. Appl. Math. 101(1-3), 167-175 (2000)

26. Kaneko, A., Kano, M.: Semi-balanced partitions of two sets of points and embeddings of rooted forests. Int. J. Comput. Geometry Appl. 15(3), 229-238 (2005)

27. Kaufmann, M., Wiese, R.: Embedding vertices at points: Few bends suffice for planar graphs. J. Graph Alg. Appl. 6(1), 115-129 (2002)

28. Kurowski, M.: A 1.235 lower bound on the number of points needed to draw all n-vertex planar graphs. Information Processing Letters 92(2), 95-98 (2004)

29. Pach, J., Wenger, R.: Embedding planar graphs at fixed vertex locations. Graphs and Combinatorics 17(4), 717-728 (2001) 